1 Hacettepe Journal of Mathematics and Statistics

$\bigcap$ Volume 46 (4) (2017), 567-577

\title{
A generalization of amenability for topological semigroups and semigroup algebras
}

\author{
M. Lashkarezadeh Bami* and H. Sadeghi ${ }^{\dagger}$
}

\begin{abstract}
In this paper for two topological semigroups $S$ and $T$, and a continuous homomorphism $\varphi$ from $S$ into $T$, we introduce and study the concept of $(\varphi, T)$-derivations on $S$ and $\varphi$-amenability of $T$ and investigate the relations between these two concepts. For two Banach algebras $A$ and $B$ and a continuous homomorphism $\varphi$ from $A$ into $B$ we also introduce the notion of $(\varphi, B)$-amenability of $A$ and show that a foundation semigroup $T$ with identity is $\varphi$-amenable whenever the Banach algebra $M_{a}(S)$ is $\left(\tilde{\varphi}, M_{a}(T)\right)$-amenable, where $\tilde{\varphi}: M(S) \longrightarrow M(T)$ denotes the unique extension of $\varphi$. An example is given to show that the converse is not true.
\end{abstract}

Keywords: Continuous homomorphism, semigroup, Banach algebra, $(\varphi, T)$-derivation, $\varphi$-amenable.

2000 AMS Classification: primary 43A07; secondary 43A10, 46H 20.

Received : 25.08.2015 Accepted : 09.08.2016 Doi : 10.15672/HJMS.20174620774

\section{Introduction}

The concept of amenability for Banach algebras was initiated by Johnson in [9]. He showed that a locally compact Hausdorff group $G$ is amenable if and only if the Banach algebra $L^{1}(G)$ is amenable. This fails to be true for discrete semigroups. Duncan and Nomioka [4] proved that if $l^{1}(S)$ is amenable then $S$ is amenable and $l^{1}(S)$ fails to be amenable if $E_{S}$ is infinite. Johonson proved that $H^{1}\left(L^{1}(G), X^{*}\right)=\{0\}$ if and only if every $G$-derivation into $X^{*}$ is inner, where $X$ is a neo-unital Banach $L^{1}(G)$-bimodule (see [2] and [9]).

Recently, Kaniuth, Lau and Pym introduced $\varphi$-amenability of a Banach algebra $A$ where $\varphi$ is a homomorphism from A to $\mathbb{C}[10]$. Here for two Banach algebras $A$ and $B$ we study the concept of $(\varphi, B)$-amenability of $A$ where $\varphi: A \longrightarrow B$ is a continuous homomorphism. In the case where $A=B, A$ is called $\varphi$-amenable. Several authors have

*M. Lashkarizadeh Bami, Email: Lashkari@sci.ui.ac.ir

$\dagger^{\dagger}$ H. Sadeghi, Email: Sadeghi@sci.ui.ac.ir 
studied $\varphi$-derivations, and $\varphi$-amenability of a Banach algebra $A$ (see [7], [8], [15] and $[16])$.

Authors in [7], introduced the notion of $\varphi$-amenability for a locally compact group $G$, where $\varphi$ is a continuous homomorphism on $G$. They proved that if the group algebra $L^{1}(G)$ is $\tilde{\varphi}$-amenable, then $G$ is $\varphi$-amenable and when $\varphi$ is an isomorphism on $G$, the converse is valid. Here $\tilde{\varphi}$ is the unique extension of $\varphi$ to $M(G)$.

In this paper for two Banach algebras $A$ and $B$ and a continuous homomorphism $\varphi$ : $A \longrightarrow B$, we first introduce the notion of $(\varphi, B)$-amenability of $A$. This concept reduces to that of $\phi$-amenability introduced by Kaniuth, Lau, and Pym, when $B=\mathbb{C}$. Also for two topological semigroups $S$ and $T$, and a continuous homomorphism $\varphi$ from $S$ into $T$, we introduce and study the concept of $(\varphi, T)$-derivations on $S$ and $\varphi$-amenability of $T$ and investigate the relation between these two concepts. Then we apply our results to the case where $S$ and $T$ are foundation semigroups and prove that $M_{a}(S)$ is $\left(\tilde{\varphi}, M_{a}(T)\right)$-amenable if and only if every $(\varphi, T)$-derivation on $S$ is $\varphi$-inner, where $\tilde{\varphi}: M_{a}(S) \longrightarrow M_{a}(T)$ denotes the extension of $\varphi$. This extends a known result due to Johonson for groups to foundation semigroups. Finally, we show that $\left(\tilde{\varphi}, M_{a}(T)\right)$-amenability of $M_{a}(S)$ implies $\varphi$-amenability of $T$, and present an example to show that the converse is not true.

\section{Preliminaries}

Let $A$ be a Banach algebra, and let $X$ be an $A$-bimodule. Then $X$ is a Banach $A$-bimodule if $X$ is a Banach space and there is a constant $k>0$ such that

$$
\|a . x\| \leq k\|a\|\|x\|, \quad\|x . a\| \leq k\|a\|\|x\| \quad(a \in A, x \in X) .
$$

By renorming, we can suppose that $k=1$. For example, $A$ itself is a Banach $A$-bimodule, and $X^{*}$, the dual space of a Banach $A$-bimodule $X$, is a Banach $A$-bimodule if for every $a \in A$ and $f \in X^{*}$ we define

$$
\langle x, a . f\rangle=\langle x . a, f\rangle, \quad\langle x, f . a\rangle=\langle a . x, f\rangle(x \in X) .
$$

We say that $X^{*}$ is the dual module of $X$.

Suppose that $A$ is a Banach algebra and $X$ is a Banach $A$-bimodule. A derivation from $A$ into $X$ is a linear operator $D: A \longrightarrow X$ satisfying

$$
D(a b)=D(a) b+a D(b)(a, b \in A) .
$$

A derivation $D$ is inner if there is $x_{0} \in X$ such that $D(a)=a . x_{0}-x_{0} . a$ for $a \in A$ and a Banach algebra $A$ is amenable if for any Banach $A$-bimodule $X$, every continuous derivation $D: A \longrightarrow X^{*}$ is inner.

Let $A$ and $B$ be two Banach algebras. The set of continuous homomorphisms from $A$ into $B$ is denoted by $\operatorname{Hom}(A, B)$. We denote the set $\operatorname{Hom}(A, A)$ by $\operatorname{Hom}(A)$.

Suppose that $\varphi: A \longrightarrow B$ is a continuous homomorphism. A Banach space $X$ over $\mathbb{C}$ is a Banach $(\varphi, B)$-bimodule if it is two-sided $\varphi(A)$-module and there is a positive real number $K$ such that

$$
\|\varphi(a) . x\| \leq K\|\varphi(a)\|_{B}\|x\| \quad\|x \cdot \varphi(a)\| \leq K\|x\|\|\varphi(a)\|_{B},
$$

for all $a \in A$ and $x \in X$.

Let $X$ be a Banach $(\varphi, B)$-bimodule and $\varphi \in \operatorname{Hom}(A, B)$, a linear operator $D: A \longrightarrow$ $X$ is called a $(\varphi, B)$-derivation if

$$
D\left(a_{1} a_{2}\right)=D\left(a_{1}\right) \cdot \varphi\left(a_{2}\right)+\varphi\left(a_{1}\right) \cdot D\left(a_{2}\right)\left(a_{1}, a_{2} \in A\right) .
$$

A $(\varphi, B)$-derivation $D$ is called $(\varphi, B)$-inner if there is $x \in X$ such that $D(a)=\varphi(a) \cdot x-$ $x . \varphi(a)(a \in A)$. A Banach algebra $A$ is called $(\varphi, B)$-amenable if for any $\operatorname{Banach}(\varphi, B)$ bimodule $X$, every continuous $(\varphi, B)$-derivation $D: A \longrightarrow X^{*}$ is $(\varphi, B)$-inner. In the case that $A=B, D$ is called $\varphi$-derivation and $A$ is called $\varphi$-amenable. 


\section{3. $\varphi$-derivation and $\varphi$-amenable semigroups}

We commence this section with the following definition:

3.1. Definition. Let $S$ and $T$ be two topological semigroups and $\varphi: S \longrightarrow T$ be a continuous homomorphism of $S$ into $T$. We say that the complex Banach space $X$ is a left Banach $\varphi$-module if there exists a mapping

$$
\varphi(S) \times X \longrightarrow X, \quad(\varphi(s), x) \longrightarrow \varphi(s) \cdot x,
$$

having the following properties:

(i) $\varphi(s) \cdot\left(x_{1}+x_{2}\right)=\varphi(s) \cdot x_{1}+\varphi(s) \cdot x_{2}, \lambda(\varphi(s) \cdot x)=\varphi(s) \cdot(\lambda x)$, $\varphi\left(s_{1} s_{2}\right) \cdot x=\varphi\left(s_{1}\right) \cdot\left(\varphi\left(s_{2}\right) \cdot x\right)$ for all $s, s_{1}, s_{2} \in S, x_{1}, x_{2} \in X$ and $\lambda \in \mathbb{C}$,

(ii) if $s_{\alpha} \longrightarrow s$ in $S$ and $x \in X$, then $\varphi\left(s_{\alpha}\right) . x \longrightarrow \varphi(s) . x$, in the norm topology, and

(iii) there is $M>0$ such that for every $x \in X$ and $s \in S$, we have

$$
\|\varphi(s) \cdot x\| \leq M\|x\| \text {. }
$$

In the same way, one defines a right Banach $\varphi$-module. The (two sided) Banach $\varphi$-module $X$ is a left and right Banach $\varphi$-module such that

$$
\left(\varphi\left(s_{1}\right) \cdot x\right) \cdot \varphi\left(s_{2}\right)=\varphi\left(s_{1}\right) \cdot\left(x \cdot \varphi\left(s_{2}\right)\right) \quad\left(s_{1}, s_{2} \in S, x \in X\right) .
$$

Note that if $X$ is a Banach $\varphi$-module, then $X^{*}$, the dual space of $X$, is also an $\varphi$-module through the following actions:

$$
\left\langle\varphi(s) \cdot x^{*}, x\right\rangle=\left\langle x^{*}, x \cdot \varphi(s)\right\rangle, \quad\left\langle x^{*} \cdot \varphi(s), x\right\rangle=\left\langle x^{*}, \varphi(s) \cdot x\right\rangle \quad\left(s \in S, x \in X, x^{*} \in X^{*}\right) .
$$

A left (resp. right) action of $\varphi(S)$ on $X$ is trivial if $\varphi(s) . x=x(s \in S, x \in X)$ (resp. $x . \varphi(s)=x(s \in S, x \in X))$.

3.2. Definition. Let $S$ and $T$ be two topological semigroups and $\varphi: S \longrightarrow T$ be a continuous homomorphism of $S$ into $T$. Let $X$ be a Banach $\varphi$-module. A weak*continuous map $D: S \longrightarrow X^{*}$ is called a $(\varphi, T)$-derivation $(\varphi$-derivation in the case that $S=T)$ if

(i) $D\left(s_{1} s_{2}\right)=\varphi\left(s_{1}\right) \cdot D\left(s_{2}\right)+D\left(s_{1}\right) \cdot \varphi\left(s_{2}\right)\left(s_{1}, s_{2} \in S\right)$;

(ii) $\sup _{s \in S}\|D(s)\|<\infty$.

Furthermore, $D$ is called $\varphi$-inner if there existe $x^{*} \in X^{*}$ such that

$$
D(s)=\varphi(s) \cdot x^{*}-x^{*} \cdot \varphi(s)(s \in S) .
$$

For a topological semigroup $S$ let $C_{b}(S)$ be the set of all bounded continuous complex valued functions on $S$ and let $\operatorname{LUC}(S)=\left\{f \in C_{b}(S) \mid x \rightarrow l_{x} f\right.$ is norm continuous $\}$ (resp. $\operatorname{RUC}(S)=\left\{f \in C_{b}(S) \mid x \rightarrow r_{x} f\right.$ is norm continuous $\left.\}\right)$, where $l_{x} f(y)=f(x y)(x, y \in S)$ (resp. $\left.r_{x} f(y)=f(y x)\right)(x, y \in S)$.

Recall that a linear functional $m \in \operatorname{LUC}(S)^{*}$ is called a mean if $\|m\|=\langle 1, m\rangle=1$; $m$ is called a left invariant mean, if $m\left(l_{s} f\right)=m(f)$ for all $s \in S$ and $f \in \operatorname{LUC}(S)$. A topological semigroup $S$ is called left amenable if $\operatorname{LUC}(S)$ has a left invariant mean (see [13] and [17]). Right amenability of a topological semigroup may be defined similarly. A topological semigroup which is both left and right amenable is called amenable.

3.3. Definition. Let $S$ and $T$ be two topological semigroups, and $\varphi: S \longrightarrow T$ be a continuous homomorphism. A $\varphi$-left invariant mean ( resp. $\varphi$-right invariant mean) on $\operatorname{LUC}(\mathrm{T})$ (resp. on $\operatorname{RUC}(\mathrm{T})$ ) is a functional $m \in \mathrm{LUC}(\mathrm{T})^{*}$ (resp. $m \in \operatorname{RUC}(\mathrm{T})^{*}$ ) such that $\langle 1, m\rangle=\|m\|=1$ and $m\left(l_{\varphi(s)} f\right)=m(f)(f \in \operatorname{LUC}(\mathrm{T}), s \in S)(\operatorname{resp} .\langle 1, m\rangle=\|m\|=1$ and $m\left(r_{\varphi(s)} f\right)=m(f)(f \in \operatorname{RUC}(\mathrm{T}), s \in S)$. 
3.4. Definition. For two topological semigroups $S$ and $T$, and continuous homomorphism $\varphi: S \longrightarrow T, T$ is called $\varphi$-left amenable ( resp. $\varphi$-right amenable) if there is a $\varphi$-left invariant mean ( resp. $\varphi$-right invariant mean) on LUC(T) (resp. on RUC(T)). A semigroup $T$ is called $\varphi$-amenable if it is both $\varphi$-left and $\varphi$-right amenable.

The proof of the following lemma is straightforward.

3.5. Lemma. Let $S$ and $T$ be two topological semigroups, and $\varphi: S \longrightarrow T$ be a continuous homomorphism. If $T$ is amenable then $T$ is $\varphi$-amenable. The converse is true if $\varphi(S)$ is dense in $T$.

3.6. Proposition. Let $S$ and $T$ be two topological semigroups, and $\varphi: S \longrightarrow T$ be a continuous homomorphism. If for every Banach $\varphi$-module $X$, any $(\varphi, T)$-derivation $D: S \longrightarrow X^{*}$ is $\varphi$-inner, then $T$ is $\varphi$-amenable.

Proof. We first note that $\operatorname{LUC}(\mathrm{T})$ is a Banach $\varphi$-module through the following actions given by

$$
\varphi(s) . f=f, \quad(f . \varphi(s))\left(t^{\prime}\right)=f\left(\varphi(s) t^{\prime}\right) \quad\left(s \in S, t^{\prime} \in T, f \in L U C(T)\right) .
$$

Let $n \in \operatorname{LUC}(\mathrm{T})^{*}$ such that $\langle 1, n\rangle=1$. Define $d: S \longrightarrow \operatorname{LUC}(\mathrm{T})^{*}$ by $d(s)=\varphi(s) \cdot n-n$. It is easy to see that $\mathbb{C} 1_{T}$ ia a closed submodule of $\operatorname{LUC}(\mathrm{T})$. Let $X=\frac{\mathrm{LUC}(\mathrm{T})}{\mathbb{C} 1_{T}}$. Since for each $s \in S,\langle 1, d(s)\rangle=0$, there exists a $(\varphi, T)$-derivation $D: S \longrightarrow X^{*}$ such that $\pi^{*} \circ D(s)=d(s)(s \in S)$, where $\pi$ is the canonical map from LUC(T) onto $X$. Thus there exists $g \in X^{*}$ such that $D(s)=\varphi(s) . g-g(s \in S)$. Hence

$$
\pi^{*} \circ D(s)=\pi^{*}(\varphi(s) \cdot g)-\pi^{*} g=\varphi(s) \cdot n-n .
$$

So

$$
\varphi(s) . n-\pi^{*}(\varphi(s) \cdot g)=n-\pi^{*} g .
$$

Let $\tilde{n}=n-\pi^{*} g$. Then $\tilde{n} \in \operatorname{LUC}(\mathrm{T})^{*}$. From (3.1), it follows that

$$
\varphi(s) \cdot \tilde{n}=\varphi(s) \cdot n-\varphi(s) \cdot\left(\pi^{*} g\right)=\varphi(s) \cdot n-\pi^{*}(\varphi(s) \cdot g)=n-\pi^{*} g=\tilde{n}(s \in S) .
$$

Since LUC(T) is a commutative $C^{*}$-algebra with identity, there exists a compact Hausdorff space $\Delta$ such that $C(\Delta)$ and $\operatorname{LUC}(\mathrm{T})$ are isometrically $*$-isomorphic $C^{*}$-algebras. Thus we can consider $\tilde{n}$ as a $\varphi$-left invariant complex Borel regular measure on $\Delta$. Let $m=\frac{|\tilde{n}|}{\|\tilde{n}\|}$, then $\varphi(s) . m=m$. Therefore for every $f \in \operatorname{LUC}(\mathrm{T})$ and $s \in S$

$$
\left\langle l_{\varphi(s)} f, m\right\rangle=\langle f . \varphi(s), m\rangle=\langle f, \varphi(s) . m\rangle=\langle f, m\rangle .
$$

Hence $T$ is $\varphi$-left amenable. Similarly we can show that $T$ is $\varphi$-right amenable. Therefore $T$ is $\varphi$-amenable

The following proposition provides a converse for Proposition 3.6 in a special case.

3.7. Proposition. Let $S$ be a topological semigroup, and $\varphi: S \longrightarrow S$ be a continuous homomorphism such that $\varphi(S)$ is dense in $S$. If $S$ is $\varphi$-left amenable, then for every Banach $\varphi$-module $X$ with trivial left action, any $\varphi$-derivation $D: S \longrightarrow X^{*}$ is $\varphi$-inner.

Proof. Suppose $S$ is $\varphi$-left amenable and $X$ is a Banach $\varphi(S)$-module with trivial left action, and $D: S \longrightarrow X^{*}$ is a $\varphi$-derivation. For every $x \in X$ we define $f_{x}: S \longrightarrow \mathbb{C}$ by $f_{x}(s)=\langle x, D(s)\rangle(s \in S)$. Thus

$$
\left\|f_{x}\right\|_{\infty}=\sup _{s \in S}\left|f_{x}(s)\right| \leq \sup _{s \in S}\|D(s)\|\|x\| \leq M\|x\|,
$$


where $M>0$ is a uniform bound for $D$, clearlly, $f_{x}$ is continiuous. We claim that $f_{x} \in \operatorname{LUC}(\mathrm{S})$. To see this let $s_{\alpha} \longrightarrow s$ in $S$, then

$$
\begin{aligned}
\left\|l_{s_{\alpha}} f_{x}-l_{s} f_{x}\right\|= & \sup _{s^{\prime} \in S}\left|f_{x}\left(s_{\alpha} s^{\prime}\right)-f_{x}\left(s s^{\prime}\right)\right| \\
= & \sup _{s^{\prime} \in S}\left|\left\langle x, D\left(s_{\alpha} s^{\prime}\right)\right\rangle-\left\langle x, D\left(s s^{\prime}\right)\right\rangle\right| \\
\leq & \sup _{s^{\prime} \in S}\left|\left\langle x, D\left(s_{\alpha}\right) \cdot \varphi\left(s^{\prime}\right)-D(s) \cdot \varphi\left(s^{\prime}\right)\right\rangle\right| \\
& +\sup _{s^{\prime} \in S}\left|\left\langle x, \varphi\left(s_{\alpha}\right) \cdot D\left(s^{\prime}\right)-\varphi(s) \cdot D\left(s^{\prime}\right)\right\rangle\right| \\
= & \left|\left\langle x, D\left(s_{\alpha}\right)-D(s)\right\rangle\right|+\sup _{s^{\prime} \in S}\left|\left\langle x \cdot \varphi\left(s_{\alpha}\right)-x \cdot \varphi(s), D\left(s^{\prime}\right)\right\rangle\right| .
\end{aligned}
$$

Since $D$ is weak*-continuous, we infer that $\left|\left\langle x, D\left(s_{\alpha}\right)-D(s)\right\rangle\right| \longrightarrow 0$. Also

$$
\sup _{s^{\prime} \in S}\left|\left\langle x . \varphi\left(s_{\alpha}\right)-x . \varphi(s), D\left(s^{\prime}\right)\right\rangle\right| \leq M\left\|x . \varphi\left(s_{\alpha}\right)-x \cdot \varphi(s)\right\|,
$$

and by definition 3.1 (ii), $\left\|x . \varphi\left(s_{\alpha}\right)-x . \varphi(s)\right\| \longrightarrow 0$. Thus $\left\|l_{t_{\alpha}} f_{x}-l_{t} f_{x}\right\| \longrightarrow 0$. So $f_{x} \in \operatorname{LUC}(\mathbf{S})$. Let $m \in \operatorname{LUC}(\mathbf{S})^{*}$ be such that $\langle 1, m\rangle=1$ and $m\left(l_{\varphi(s)} f\right)=m(f)(s \in S, f \in$ LUC(S)), and define a linear functional $f$ on $X$ by $\langle x, f\rangle=\left\langle f_{x}, m\right\rangle(x \in X)$. For every $x \in X$ and $s, s^{\prime} \in S$, we have

$$
\begin{aligned}
f_{x . \varphi(s)}\left(s^{\prime}\right) & =\left\langle x \cdot \varphi(s), D\left(s^{\prime}\right)\right\rangle=\left\langle x, \varphi(s) \cdot D\left(s^{\prime}\right)\right\rangle \\
& =\left\langle x, D\left(s s^{\prime}\right)\right\rangle-\left\langle x, D(s) \cdot \varphi\left(s^{\prime}\right)\right\rangle \\
& =\left\langle x, D\left(s s^{\prime}\right)\right\rangle-\langle x, D(s)\rangle \\
& =f_{x}\left(s s^{\prime}\right)-\langle x, D(s)\rangle 1_{S}\left(s^{\prime}\right) .
\end{aligned}
$$

Therefore $f_{x . \varphi(s)}=l_{s} f_{x}-\langle x, D(s)\rangle 1_{S}$. Since $\varphi(S)$ is dense in $S$ it follows that there exists a net $\left\{s_{\alpha}\right\}$ in $S$ such that $\lim _{\alpha} \varphi\left(s_{\alpha}\right)=s$, and $\lim _{\alpha} l_{\varphi\left(s_{\alpha}\right)} f_{x}=l_{s} f_{x}$ by the definition of $L U C(S)$. Thus

$$
\begin{aligned}
\langle x, f-\varphi(s) . f\rangle & =\langle x, f\rangle-\langle x . \varphi(s), f\rangle=\left\langle f_{x}-f_{x . \varphi(s)}, m\right\rangle \\
& =\left\langle f_{x}-l_{s} f_{x}+\langle x, D(s)\rangle 1_{S}, m\right\rangle \\
& =\left\langle f_{x}-\lim _{\alpha} l_{\varphi\left(s_{\alpha}\right)} f_{x}+\langle x, D(s)\rangle 1_{S}, m\right\rangle \\
& =\lim _{\alpha}\left\langle f_{x}-l_{\varphi\left(s_{\alpha}\right)} f_{x}+\langle x, D(s)\rangle 1_{S}, m\right\rangle \\
& =\langle x, D(s)\rangle .
\end{aligned}
$$

Hence $D(s)=f-\varphi(s) . f(s \in S)$. Therefore $D$ is $\varphi$-inner.

By a similar argument one can prove the following proposition:

3.8. Proposition. Let $S$ be topological semigroup, and $\varphi: S \longrightarrow S$ be a continuous homomorphism such that $\varphi(S)$ is dense in $S$. If $S$ is $\varphi$-right amenable, then for every Banach $\varphi$-module $X$ with trivial right action, any $\varphi$-derivation $D: S \longrightarrow X^{*}$ is $\varphi$-inner.

\section{4. $\tilde{\varphi}$ - amenability of $M_{a}(S)$}

We start this section with the following.

For a topological semigroup $S$ let $M(S)$ denote the space of all bounded, regular, complex Borel measures on $S$. This space with the convolution product and norm $\|\mu\|=$ $|\mu|(S)$ is a Banach algebra. The space of all measures $\mu \in M(S)$ for which the mappings $x \rightarrow|\mu| * \delta_{x}$, and $x \rightarrow \delta_{x} *|\mu|$ from $S$ into $M(S)$ are weakly continuous is denote by $M_{a}(S)(L(S)$, as in [1]). A Hausdorff locally compact topological semigroup $\mathrm{S}$ is called a foundation semigroup if $S$ coincides with the closure of $\bigcup\left\{\operatorname{supp}(\mu): \mu \in M_{a}(S)\right\}$. Note 
that in the case where $\mathrm{S}$ is a foundation semigroup with identity, for every $\mu \in M_{a}(S)$ both mappings $x \rightarrow|\mu| * \delta_{x}$, and $x \rightarrow \delta_{x} *|\mu|$ from $S$ into $M(S)$ are norm continuous and $M_{a}(S)$ has a bounded approximate identity(see [6]).

Finally, for any topological semigroup $S, \mu \in M(S)$ and $f \in C_{b}(S)$, we define the complex valued funcions $\mu \circ f$ and $f \circ \mu$ on $S$ by

$$
\left.\mu \circ f(x)=\int_{S} f(y x)\right) d \mu(y), \quad f \circ \mu(x)=\int_{S} f(x y) d \mu(y) .
$$

Lemma 1.3.4 of [6], shows that $\mu \circ f$ and $f \circ \mu$ are in $C_{b}(S)$. Also for every $\mu, \nu \in M_{a}(S)$ and $f \in C_{b}(S)\langle\mu * \nu, f\rangle=\langle\mu, f \circ \nu\rangle=\langle\nu, \mu \circ f\rangle$.

4.1. Definition. If a Banach algebra $A$ is contained in a Banach algebra $B$ as a closed ideal, then the strict topology or strong operator topology (so) on $B$ with respect to $A$ is defined through the family of seminorms $\left(p_{a}\right)_{a \in A}$, where

$$
p_{a}(b):=\|b a\|+\|a b\| \quad(b \in B) .
$$

For a topological semigroup $S$ the strict topology on $M(S)$ with respect to $M_{a}(S)$ is simply called so topology or the strict topology on $M(S)$.

4.2. Lemma. Let $S$ and $T$ be two foundation semigroups with identity, and let $\varphi: S \longrightarrow$ $T$ be a continuous homomorphism. Define $\tilde{\varphi}: M(S) \longrightarrow M(T)$ by

$$
\langle\tilde{\varphi}(\mu), f\rangle=\int_{S} f(\varphi(x)) d \mu(x) \quad\left(f \in C_{0}(T)\right) .
$$

Then $\tilde{\varphi}$ is a continuous homomorphism (with respect to the strict topology on $M(S)$ ) that extends $\varphi$ uniquely and $\tilde{\varphi}\left(M_{a}(S)\right) \subseteq M_{a}(T)$.

Proof. It is easy to see that $\tilde{\varphi}$ is continuous. By using (4.1), for every $f \in C_{0}(T)$ and $\mu_{1}, \mu_{2} \in M(S)$, we have

$$
\begin{aligned}
\left\langle\tilde{\varphi}\left(\mu_{1}\right) * \tilde{\varphi}\left(\mu_{2}\right), f\right\rangle & =\left\langle\tilde{\varphi}\left(\mu_{1}\right), f \circ \tilde{\varphi}\left(\mu_{2}\right)\right\rangle \\
& =\int_{S} f \circ \tilde{\varphi}\left(\mu_{2}\right)(\varphi(x)) d \mu_{1}(x) \\
& =\int_{S} \int_{S} f(\varphi(x) y) d \tilde{\varphi}\left(\mu_{2}\right)(y) d \mu_{1}(x) \\
& =\int_{S} \int_{S} f(\varphi(x) \varphi(y)) d \mu_{2}(y) d \mu_{1}(x) \\
& =\int_{S} \int_{S} f(\varphi(x) \varphi(y)) d \mu_{1}(x) d \mu_{2}(y) \\
& =\int_{S} f(\varphi(x)) d \mu_{1} * \mu_{2}(x) \\
& =\left\langle\tilde{\varphi}\left(\mu_{1} * \mu_{2}\right), f\right\rangle .
\end{aligned}
$$

Therefore $\tilde{\varphi}$ is a continuous homomorphism. Let $\bar{\varphi}$ be another extension of $\varphi$ and let $\mu \in M(S)$. By Theorem 3.3 of [14], $\mu$ is the s-lim (strict-lim) of a net $\left(\mu_{i}\right)$ such that each $\mu_{i}$ is a combination of point masses. So,

$$
\tilde{\varphi}(\mu)=\tilde{\varphi}\left(s-\lim _{i} \mu_{i}\right)=\lim _{i} \tilde{\varphi}\left(\mu_{i}\right)=\lim _{i} \bar{\varphi}\left(\mu_{i}\right)=\bar{\varphi}\left(s-\lim _{i} \mu_{i}\right)=\bar{\varphi}(\mu) .
$$

Thus $\tilde{\varphi}=\bar{\varphi}$.

To complete the prove, let $\left(\mu_{\alpha}\right)$ be a bounded approximate identity for $M_{a}(T)$, then as in Lemma 2.1 of [11],

$$
\left\|\mu_{\alpha} \circ f-f\right\|_{\infty} \longrightarrow 0 \quad\left(f \in C_{0}(T)\right) .
$$


Now for every $\mu \in M_{a}(T)$, we obtain

$$
\begin{aligned}
\left\|\tilde{\varphi}(\mu) * \mu_{\alpha}-\tilde{\varphi}(\mu)\right\| & =\sup _{f \in C_{0}(T),\|f\|_{\infty} \leq 1}\left|\left\langle\tilde{\varphi}(\mu) * \mu_{\alpha}, f\right\rangle-\langle\tilde{\varphi}(\mu), f\rangle\right| \\
& =\sup _{f \in C_{0}(T),\|f\|_{\infty} \leq 1}\left|\left\langle\tilde{\varphi}(\mu), \mu_{\alpha} \circ f\right\rangle-\langle\tilde{\varphi}(\mu), f\rangle\right| \\
& =\sup _{f \in C_{0}(T),\|f\|_{\infty} \leq 1}\left|\left\langle\tilde{\varphi}(\mu), \mu_{\alpha} \circ f-f\right\rangle\right| \\
& \leq \sup _{f \in C_{0}(T),\|f\|_{\infty} \leq 1}\|\tilde{\varphi}(\mu)\|\left\|\mu_{\alpha} \circ f-f\right\|_{\infty} \longrightarrow 0 .
\end{aligned}
$$

This means that $\tilde{\varphi}(\mu) * \mu_{\alpha} \longrightarrow \tilde{\varphi}(\mu)$ in norm. So $\tilde{\varphi}(\mu) \in M_{a}(T)$. Therefore $\tilde{\varphi}\left(M_{a}(S)\right) \subseteq$ $M_{a}(T)$.

4.3. Definition. Let $A$ and $B$ be two Banach algebras and $\varphi: A \longrightarrow B$ be a continuous homomorphism. A Banach $(\varphi, B)$-bimodule $X$ is called $\varphi$-pseudo-unital if

$$
X=\left\{\varphi\left(a_{1}\right) \cdot x \cdot \varphi\left(a_{2}\right): a_{1}, a_{2} \in A, x \in X\right\} .
$$

The proof of the following proposition is omitted, since it can be proved in the same direction of Proposition 2.1.3 of [18].

4.4. Proposition. Let $A$ and $B$ be two Banach algebras which $A$ has a bounded right approximate identity, and $\varphi: A \longrightarrow B$ be a continuous homomorphism. Let $X$ be a Banach $(\varphi, B)$-bimodule such that $\varphi(A) \cdot X=\{0\}$. Then every $(\varphi, B)$-derivation on $A$ is $(\varphi, B)$-inner.

Similarly, we can proof above proposition for a Banach algebra $A$ with a bounded left approximate identity, where the module action from the right is trivial.

By using above proposition and similar argument as in the proof of the Proposition 2.1.5 of [18], we can proof following proposition.

4.5. Proposition. Let $A$ and $B$ be two Banach algebras with bounded approximate identity, and $\varphi: A \longrightarrow B$ be a continuous homomorphism. Then the following two condition are equivalent:

(i) For each Banach $(\varphi, B)$-bimodule $X$, any continuous $(\varphi, B)$-derivation on $A$ is $(\varphi, B)$-inner.

(ii) For each $\varphi$-pseudo-unital Banach $(\varphi, B)$-bimodule $X$, any continuous $(\varphi, B)$ derivation on $A$ is $(\varphi, B)$-inner.

The following proposition generalizes Proposition 2.1.6 of [18].

4.6. Proposition. Let $A_{1}$ and $A_{2}$ be two Banach algebras with bounded approximate identity which are closed ideals of Banach algebras $B_{1}$ and $B_{2}$, respectively. Let $\varphi$ : $A_{1} \longrightarrow A_{2}$ be a continuous homomorphism and $X$ be a $\varphi$-pseudo-unital Banach $\left(\varphi, A_{2}\right)$ bimodule, and $\tilde{\varphi}: B_{1} \longrightarrow B_{2}$ be a continuous homomorphism such that $\left.\tilde{\varphi}\right|_{A_{1}}=\varphi$. Let $D: A_{1} \longrightarrow X^{*}$ be a $\left(\varphi, A_{2}\right)$-derivation, then $X$ is a Banach $\left(\tilde{\varphi}, B_{2}\right)$-bimodule and there is a unique $\left(\tilde{\varphi}, B_{2}\right)$ derivation $\tilde{D}: B_{1} \longrightarrow X^{*}$ satisfying the following:

(i) $\left.\tilde{D}\right|_{A_{1}}=D$;

(ii) $\tilde{D}$ is continuous with respect to the strict topology on $B_{1}$ and the $w^{*}$-topology on $X^{*}$.

Proof. For $x \in X$, let $\varphi\left(a_{1}\right) \in A_{2}$ and $y \in X$ be such that $x=\varphi\left(a_{1}\right)$.y. For $b_{1} \in B_{1}$, define $\tilde{\varphi}\left(b_{1}\right) \cdot x:=\tilde{\varphi}\left(b_{1} a_{1}\right) \cdot y$. We claim that $\tilde{\varphi}\left(b_{1}\right) \cdot x$ is well define. Let $\varphi\left(a_{1}^{\prime}\right) \in A_{2}$ and 
$y^{\prime} \in X$ be such that $x=\varphi\left(a_{1}^{\prime}\right) \cdot y^{\prime}$, and let $\left(f_{\beta}\right)_{\beta}$ be a bounded approximate identity for $A_{2}$. Then

$$
\tilde{\varphi}\left(b_{1} a_{1}\right) \cdot y=\lim _{\beta} \tilde{\varphi}\left(b_{1}\right) f_{\beta} \varphi\left(a_{1}\right) \cdot y=\lim _{\beta} \tilde{\varphi}\left(b_{1}\right) f_{\beta} \varphi\left(a_{1}^{\prime}\right) \cdot y^{\prime}=\tilde{\varphi}\left(b_{1} a_{1}^{\prime}\right) \cdot y^{\prime}\left(b_{1} \in B_{1}\right) .
$$

It is obvious that this operation of $\tilde{\varphi}\left(B_{1}\right)$ on $X$ turns $X$ into a left $\tilde{\varphi}\left(B_{1}\right)$-bimodule. Similarly, one defines a right Banach $\tilde{\varphi}\left(B_{1}\right)$-module structure on $X$, so that $X$ becomes a Banach $\left(\tilde{\varphi}, B_{2}\right)$-bimodule. Now we define $\tilde{D}: B_{1} \longrightarrow X^{*}$ by

$$
\tilde{D}\left(b_{1}\right)=w^{*}-\lim _{\alpha}\left(D\left(b_{1} e_{\alpha}\right)-\tilde{\varphi}\left(b_{1}\right) \cdot D\left(e_{\alpha}\right)\right),
$$

where $\left(e_{\alpha}\right)_{\alpha}$ is a bounded approximate identity for $A_{1}$. By the similar argument as in the proof of Proposition 3.1 of [7], one can show that $\tilde{D}$ is define a $\varphi$-derivation on $B_{1}$ where $\left.\tilde{D}\right|_{A_{1}}=D$ and $\tilde{D}$ is continuous with respect to the strict topology on $B_{1}$ and the w*-topology on $X^{*}$.

4.7. Theorem. Let $S$ and $T$ be two foundation semigroups with identity, and $\varphi: S \longrightarrow T$ be a continuous homomorphism, and $\tilde{\varphi}$ be as in Lemma 4.2. Then $M_{a}(S)$ is $\left(\tilde{\varphi}, M_{a}(T)\right)$ amenable if and only if every $(\varphi, T)$-derivation on $S$ is $\varphi$-inner.

Proof. Suppose $M_{a}(S)$ is $\left(\tilde{\varphi}, M_{a}(T)\right)$-amenable, and $D: S \longrightarrow X^{*}$ is a $(\varphi, T)$-derivation on $S$ for some $\varphi$-module $X$. For every $\mu \in M_{a}(S)$ and $x \in X$ we define

$$
\tilde{\varphi}(\mu) \cdot x=\int_{\varphi(S)} t \cdot x d \tilde{\varphi}(\mu)(t), \quad x \cdot \tilde{\varphi}(\mu)=\int_{\varphi(S)} x \cdot t d \tilde{\varphi}(\mu)(t) .
$$

So for some $k>0$,

$$
\int_{\varphi(S)}\|t . x\| d|\tilde{\varphi}(\mu)|(t) \leq k\|x\|\|\tilde{\varphi}(\mu)\|<\infty
$$

Therefore $\tilde{\varphi}(\mu) \cdot x$ is well defined with $\|\tilde{\varphi}(\mu) \cdot x\| \leq k\|\tilde{\varphi}(\mu)\|\|x\|$. Similarly one can define $x . \tilde{\varphi}(\mu)$ satisfying $\|x . \tilde{\varphi}(\mu)\| \leq k\|x\|\|\tilde{\varphi}(\mu)\|$. Thus $X$ defines a Banach $\left(\tilde{\varphi}, M_{a}(T)\right)$ bimodule. Using (4.3) and the definition of $\tilde{\varphi}$ we obtain

$$
\tilde{\varphi}(\mu) \cdot x=\int_{S} \varphi(s) \cdot x d \mu(s), \quad x \cdot \tilde{\varphi}(\mu)=\int_{S} x \cdot \varphi(s) d \mu(s),
$$

for all $x \in X, \mu \in M_{a}(S)$. Define $\tilde{D}: M_{a}(S) \longrightarrow X^{*}$ by

$$
\langle x, \tilde{D}(\mu)\rangle=\int_{S}\langle x, D(s)\rangle d \mu(s) \quad\left(\mu \in M_{a}(S), x \in X\right) .
$$


Clearly, $\tilde{D}$ is continuous. By using (4.4), for every $\mu_{1}, \mu_{2} \in M_{a}(S)$ and $x \in X$, we have

$$
\begin{aligned}
\left\langle x, \tilde{D}\left(\mu_{1} * \mu_{2}\right)\right\rangle= & \int_{S}\langle x, D(s)\rangle d \mu_{1} * \mu_{2}(s) \\
= & \int_{S} \int_{S}\left\langle x, D\left(s_{1} s_{2}\right)\right\rangle d \mu_{1}\left(s_{1}\right) d \mu_{2}\left(s_{2}\right) \\
= & \int_{S} \int_{S}\left\langle x, D\left(s_{1}\right) \cdot \varphi\left(s_{2}\right)+\varphi\left(s_{1}\right) \cdot D\left(s_{2}\right)\right\rangle d \mu_{1}\left(s_{1}\right) d \mu_{2}\left(s_{2}\right) \\
= & \int_{S} \int_{S}\left\langle\varphi\left(s_{2}\right) \cdot x, D\left(s_{1}\right)\right\rangle d \mu_{1}\left(s_{1}\right) d \mu_{2}\left(s_{2}\right) \\
& +\int_{S} \int_{S}\left\langle x \cdot \varphi\left(s_{1}\right), D\left(s_{2}\right)\right\rangle d \mu_{1}\left(s_{1}\right) d \mu_{2}\left(s_{2}\right) \\
= & \int_{S}\left\langle\int_{S} \varphi\left(s_{2}\right) \cdot x d \mu_{2}\left(s_{2}\right), D\left(s_{1}\right)\right\rangle d \mu_{1}\left(s_{1}\right) \\
& +\int_{S}\left\langle\int_{S} x \cdot \varphi\left(s_{1}\right) d \mu_{1}\left(s_{1}\right), D\left(s_{2}\right)\right\rangle d \mu_{2}\left(s_{2}\right) \\
= & \int_{S}\left\langle\tilde{\varphi}\left(\mu_{2}\right) \cdot x, D\left(s_{1}\right)\right\rangle d \mu_{1}\left(s_{1}\right)+\int_{S}\left\langle x \cdot \tilde{\varphi}\left(\mu_{1}\right), D\left(s_{2}\right)\right\rangle d \mu_{2}\left(s_{2}\right) \\
= & \left\langle\tilde{\varphi}\left(\mu_{2}\right) \cdot x, \tilde{D}\left(\mu_{1}\right)\right\rangle+\left\langle x \cdot \tilde{\varphi}\left(\mu_{1}\right), \tilde{D}\left(\mu_{2}\right)\right\rangle \\
= & \left\langle x, \tilde{D}\left(\mu_{1}\right) \cdot \tilde{\varphi}\left(\mu_{2}\right)+\tilde{\varphi}\left(\mu_{1}\right) \cdot \tilde{D}\left(\mu_{2}\right)\right\rangle .
\end{aligned}
$$

That is $\tilde{D}\left(\mu_{1} * \mu_{2}\right)=\tilde{D}\left(\mu_{1}\right) \cdot \tilde{\varphi}\left(\mu_{2}\right)+\tilde{\varphi}\left(\mu_{1}\right) \cdot \tilde{D}\left(\mu_{2}\right)$. Hence $\tilde{D}$ is a $\left(\tilde{\varphi}, M_{a}(T)\right)$-derivation. From the $\left(\tilde{\varphi}, M_{a}(T)\right)$-amenability of $M_{a}(S)$ it follows that there exists $x^{*} \in X^{*}$ such that $\tilde{D}(\mu)=\tilde{\varphi}(\mu) \cdot x^{*}-x^{*} \cdot \tilde{\varphi}(\mu)\left(\mu \in M_{a}(S)\right)$. Moreover, for every $x \in X$ and $\mu \in M_{a}(S)$, we have

$$
\begin{aligned}
\int_{S}\langle x, D(s)\rangle d \mu(s) & =\langle x, \tilde{D}(\mu)\rangle \\
& =\left\langle x, \tilde{\varphi}(\mu) \cdot x^{*}-x^{*} \cdot \tilde{\varphi}(\mu)\right\rangle \\
& =\left\langle x, \tilde{\varphi}(\mu) \cdot x^{*}\right\rangle-\left\langle x, x^{*} \cdot \tilde{\varphi}(\mu)\right\rangle \\
& =\left\langle x, \int_{S} \varphi(s) \cdot x^{*} d \mu(s)\right\rangle-\left\langle x, \int_{S} x^{*} \cdot \varphi(s) d \mu(s)\right\rangle \\
& =\int_{S}\left\langle x, \varphi(s) \cdot x^{*}-x^{*} \cdot \varphi(s)\right\rangle d \mu(s) .
\end{aligned}
$$

So $\langle x, D(s)\rangle=\left\langle x, \varphi(s) \cdot x^{*}-x^{*} \cdot \varphi(s)\right\rangle(x \in X)$, by Lemma 2.2 of [12]. Hence

$$
D(s)=\varphi(s) \cdot x^{*}-x^{*} \cdot \varphi(s)(s \in S) \text {. }
$$

Therefore $D$ is $\varphi$-inner.

Conversely, suppose every $(\varphi, T)$-derivation on $S$ is $\varphi$-inner. Let $D: M_{a}(S) \longrightarrow X^{*}$ be a $\left(\tilde{\varphi}, M_{a}(T)\right)$-derivation for some Banach $\left(\tilde{\varphi}, M_{a}(T)\right)$-bimodule $X$. By Proposition 4.5, there is no loss of generality if we suppose that $X$ is $\tilde{\varphi}$-pseudo-unital. So by Proposition 4.6, $X$ is a Banach $(\tilde{\varphi}, M(T))$-bimodule and there is a unique $(\tilde{\varphi}, M(T))$-derivation $\tilde{D}$ : $M(S) \longrightarrow X^{*}$ that extends $D$ and is continuous with respect to the strict topology on $M(S)$ and the $w^{*}$-topology on $X^{*}$. We consider the following module actions $\varphi(S)$ on $X$ by

$$
\varphi(s) . x:=\delta_{\varphi(s)} \cdot x, \quad x . \varphi(s):=x . \delta_{\varphi(s)} \quad(s \in S, x \in X),
$$

and define $D_{S}: S \longrightarrow X^{*}$ by $D_{S}(s)=\tilde{D}\left(\delta_{s}\right)(s \in S)$. It is easy to see that $D_{S}$ defines a $(\varphi, T)$-derivation. So there exists $x^{*} \in X^{*}$ such that

$$
D_{S}(s)=\varphi(s) \cdot x^{*}-x^{*} \cdot \varphi(s)(s \in S) .
$$


Consequently, for every $s \in S$

$$
\tilde{D}\left(\delta_{s}\right)=D_{S}(s)=\varphi(s) \cdot x^{*}-x^{*} \cdot \varphi(s)=\tilde{\varphi}\left(\delta_{s}\right) \cdot x^{*}-x^{*} \cdot \tilde{\varphi}\left(\delta_{s}\right) .
$$

Since every measure $\mu$ in $M(S)$ is the s-lim of a net $\left(\mu_{i}\right)$ such that each $\mu_{i}$ is a combination of point masses (see Theorem 3.3 of [14]), from the definition of the strict topology it follows that $\nu * \mu_{i} \longrightarrow \nu * \mu\left(\nu \in M_{a}(S)\right)$ and $\mu_{i} * \nu \longrightarrow \mu * \nu\left(\nu \in M_{a}(S)\right)$ in the norm topology. Let $x \in X$, and $\tilde{\varphi}(\nu) \in M_{a}(T)$ and $y \in X$ be such that $x=y \cdot \tilde{\varphi}(\nu)$. Hence

$$
\begin{aligned}
\left|\left\langle x, \tilde{\varphi}\left(\mu_{i}\right) \cdot x^{*}\right\rangle-\left\langle x, \tilde{\varphi}(\mu) \cdot x^{*}\right\rangle\right| & =\left|\left\langle y \cdot \tilde{\varphi}(\nu), \tilde{\varphi}\left(\mu_{i}\right) \cdot x^{*}\right\rangle-\left\langle y \cdot \tilde{\varphi}(\nu), \tilde{\varphi}(\mu) \cdot x^{*}\right\rangle\right| \\
& =\left|\left\langle y \cdot \tilde{\varphi}\left(\nu * \mu_{i}\right)-y \cdot \tilde{\varphi}(\nu * \mu), x^{*}\right\rangle\right| \\
& \leqslant k\|y\|\|\tilde{\varphi}\|\left\|\nu * \mu_{i}-\nu * \mu\right\|\left\|x^{*}\right\| \longrightarrow 0 .
\end{aligned}
$$

This means that $w^{*}-\lim _{i} \tilde{\varphi}\left(\mu_{i}\right) \cdot x^{*}=\tilde{\varphi}(\mu) \cdot x^{*}$. Similarly, $w^{*}-\lim _{i} x^{*} \cdot \tilde{\varphi}\left(\mu_{i}\right)=x^{*} \cdot \tilde{\varphi}(\mu)$. Now for every $\mu \in M(S)$, we obtain

$$
\begin{aligned}
\tilde{D}(\mu) & =\tilde{D}\left(s-\lim _{i} \mu_{i}\right)=w^{*}-\lim _{i} \tilde{D}\left(\mu_{i}\right) \\
& =w^{*}-\lim _{i}\left(\tilde{\varphi}\left(\mu_{i}\right) \cdot x^{*}-x^{*} \cdot \tilde{\varphi}\left(\mu_{i}\right)\right) \\
& =\tilde{\varphi}(\mu) \cdot x^{*}-x^{*} \cdot \tilde{\varphi}(\mu) .
\end{aligned}
$$

Thus $\tilde{D}$ is a $(\tilde{\varphi}, M(T))$-inner derivation and so $D$ is $\left(\tilde{\varphi}, M_{a}(T)\right)$-inner derivation. Therefore $M_{a}(S)$ is $\left(\tilde{\varphi}, M_{a}(T)\right)$-amenable.

A combination of Proposition 3.6 and Theorem 4.7, gives the following result.

4.8. Theorem. Let $S$ and $T$ be two foundation semigroups with identity, and let $\varphi$ : $S \longrightarrow T$ be a continuous homomorphism, and $\tilde{\varphi}$ be as in Lemma 4.2. If $M_{a}(S)$ is $\left(\tilde{\varphi}, M_{a}(T)\right)$-amenable, then $T$ is $\varphi$-amenable.

Before turning the next result, we first need to prove the following proposition.

4.9. Proposition. Let $A$ and $B$ be two Banach algebras and let $\varphi: A \longrightarrow B$ be a continuous homomorphism. If $\varphi(A)$ is dense in $B$ and $A$ is $(\varphi, B)$-amenable, then $B$ is amenable.

Proof. Let $D: B \longrightarrow X^{*}$ be a continuous derivation for a Banach $B$-bimodule $X$, and $\tilde{D}=D \circ \varphi$. Obviously $\tilde{D}$ define a continuous $(\varphi, B)$-derivation from $A$ into $X^{*}$. By $(\varphi, B)$-amenability of $A$ there exists $f \in X^{*}$ such that

$$
\tilde{D}(a)=\varphi(a) \cdot f-f \cdot \varphi(a)(a \in A) .
$$

Let $b \in B$, since $\varphi(A)$ is dense in $B$, there exists a net $\left\{a_{\alpha}\right\} \subset A$ such that $\lim _{\alpha} \varphi\left(a_{\alpha}\right)=b$. Hence

$$
D(b)=\lim _{\alpha} D\left(\varphi\left(a_{\alpha}\right)\right)=\lim _{\alpha} \tilde{D}\left(a_{\alpha}\right)=\lim _{\alpha}\left(\varphi\left(a_{\alpha}\right) . f-f . \varphi\left(a_{\alpha}\right)\right)=b . f-f . b .
$$

Thus $D$ is an inner derivation. This completes the proof.

Note that if $S$ is a discrete semigroup then $S$ is a foundation semigroup with $\operatorname{LUC}(S)=$ $l^{\infty}(S)$, and $M(S)=M_{a}(S)=l^{1}(S)$.

The next example shown that the converses of the Theorem 4.8 and Proposition 3.6 are not true.

4.10. Example. Let $S$ be the set $\mathbb{Z}=\{\cdots,-2,-1,0,1,2, \cdots\}$, with the product

$$
(m, n) \longrightarrow m \vee n=\max \{m, n\}, \quad \mathbb{Z} \times \mathbb{Z} \longrightarrow \mathbb{Z} .
$$

Define $\varphi: S \longrightarrow S$ by $\varphi(s)=s+1(s \in S)$. Then it is easy to check that $\varphi$ is a homomorphism on $S$. Since $S$ is an abelian semigroup it follows $S$ is amenable (see [3]) and so by Lemma 3.5, $S$ is $\varphi$-amenable. Since $E(S)=S$, from Corollary 1 of [5], the 
convolution semigroup algebra $l^{1}(S)$ is not amenable. Also since $\varphi$ is an epimorphism on $S$ it follows that $\tilde{\varphi}$ is an epimorphism on $l^{1}(S)$. Therefore by Proposition $4.9, l^{1}(S)$ is not $\tilde{\varphi}$-amenable. So the converse of the Theorem 4.8 is not valid. Also by Theorem 4.7 , we conclude that the converse of the Proposition 3.6 is not true.

Acknowledgement. We are thankful to the referees for their valuable suggestions and comments and grateful to the office of Graduate studies of the university of Isfahan for their support.

\section{References}

1. A. C. Baker, J. W. Baker, Algebra of measures on a locally compact semigroup III, J. London Math. Soc. (4), 685-695, 1972.

2. H. G. Dales, Banach slgebras and automatic continuity, Clarendon Press, Oxford, 2000.

3. M. M. Day, Ergodic theorems for Abelian semigroups, Trans. Amer. Math. Soc., 51, 399-412, 1972.

4. J. Duncan, I. Namioka, Amenability of inverse semigroups and thier semigroup algebras, Proceeding of the Royal Society of Edinburgh 80 A, 309-321, 1998.

5. J. Duncan, A. L. T. Paterson, Amenability for discrete convolution semigroup algebras, Math. Scand. 66, 141-146, 1990.

6. H. A. M. Dzinotyiweyi, The analoge of the group algebra for topological semigroups, Research Notes in Mathematics, 98, Pitman, New York, 1984.

7. Z. Ghorbani, M. Lashkarizadeh Bami, $\varphi$-Approximate biflat and $\varphi$-amenabel Banach algebras, Proceedings of the Romanian Academy, Series A, 13 (1), 3-10, 2012.

8. Z. Ghorbani, M. Lashkarizadeh Bami, $\varphi$-Amenable and $\varphi$-biflat Banach algebras, Bull. Iranian Math. Soc. 39 (3), 507-515, 2013.

9. B. E. Johnson, Cohomology in Banach algebras, harmonic problems, Memoirs Amer. Math. Soc. 127, 1972.

10. E. Kaniuth, A. Lau, J. Pym, On $\varphi$-amenability of Banach algebras, Math. Proc. Camp. Phil. Soc., 144, 85-96, 2008.

11. M. Lashkarizadeh Bami, Ideals of $\mathrm{M}(\mathrm{S})$ as ideals of $L U C(S)^{*}$ of a compactly cancellative semigroup S, Math. Japon., 48, 363-366, 1998.

12. M. Lashkarizadeh Bami, Representations of foundation semigroups and their algebras, Canadian J. Math, 37, 29-47, 1985.

13. A. T. M. Lau, Amenability of semigroups, the analytical and topological theory of semigroups, trends and developments K. H. Hofman, J. D. Lawson and J. S. Pym, eds., Walter de Gruyter and Co., 1990.

14. M. Lashkarizadeh Bami, B. Mohammadzadeh and H. Samea, Derivations on certain semigroup algebras, Journal of Sciences Islamic Republic of Iran, 18 (4), 339-345, 2007.

15. M. Mirzavaziri and M. S. Moslehian, $\sigma$-derivations in Banach algebras, Bull. Iranian Math. Soc. 32 (1), 65-78, 2006.

16. M. Mirzavaziri and M.S. Moslehian, Automatic continuity of $\sigma$-derivations in $C^{*}$-algebras, Proc. Amer. Math. Soc. 134 (11), 3319-3327, 2006.

17. I. Namioka, On certain actions of semigroups on L-spaces, Studia Math, 29, 63-77, 1967.

18. V. Runde, Lectures on Amenability, Lecture Notes in Mathematics 1774, Springer-Verlag, Berlin-Heidelberg-New York, 2002. 
